ISSN: 1676 - 1901

\title{
APLICAÇÃO DE UM MODELO MATEMÁTICO NA SIMULAÇÃO DA PRODUÇÃO E IMPORTAÇÃO DE GÁS NATURAL NO BRASIL ATÉ 2017
}

\author{
APPLICATION OF A MATHEMATICAL MODEL TO SIMULATE THE \\ PRODUCTION AND IMPORT OF NATURAL GAS IN BRAZIL UNTIL 2017
}

\author{
Antonio Carlos Gracias* antonio.gracias@ufabc.edu.br \\ Sérgio Ricardo Lourenço* sergio.lourenco@ufabc.edu.br \\ * Universidade Federal do ABC
}

\begin{abstract}
Resumo: $O$ governo federal, por meio de política de desenvolvimento, pretende ampliar a participação do gás natural na matriz energética nos próximos anos. Essa ampliação irá produzir um aumento da capacidade de abastecimento de energia elétrica com uso da geração de energia térmica com gás natural. Um modelo matemático capaz de possibilitar uma previsão futura do consumo e importação de gás natural é fundamental para o planejamento da matriz energética do Brasil. Este trabalho compara dois modelos matemáticos, o modelo de Verhulst (modelo logístico) e o modelo de Malthus (modelo exponencial), com o objetivo de analisar as possibilidades desses modelos descreverem a evolução da produção e importação de gás natural no Brasil até 2017, a partir dos dados fornecidos pelo Balanço Energético Nacional desde 1970 até 2007. Toda parte computacional, gráficos, resolução das equações diferenciais e cálculos de linearização e método dos mínimos quadrados, foi feita com o uso do software MatLab ${ }^{\circledR}$. Os resultados obtidos, por meio de gráficos, mostram que os modelos de dinâmica populacional (Verhulst e Malthus) podem ser aplicados na modelagem da produção e importação de gás natural do Brasil.
\end{abstract}

Palavras-chave: Modelo de Malthus. Modelo de Verhulst. Modelagem matemática. Gás natural.

Abstract. The Brazilian federal government, through policy of development, intends to increase the participation of natural gas in the energy matrix in the coming years. This expansion will generate an increase in the supply of electricity by heat generation through natural gas. A mathematical model capable of providing a forecast of future consumption and import of natural gas is essential to the planning of the Brazilian energy matrix. This study aims to compare two mathematical models, Verhulst's model (logistic model) and Malthus's model (exponential model) to analyze the possibilities of these models to describe the evolution of production and import of natural gas in Brazil, from 1970 to 2007. All the computer work used in this article: graphics, resolution of differential equations, calculations of linearization and the least squares fitting was prepared in the software MatLab. The results obtained through graphs showed that the model of Verhulst for import gas and the model of Malthus for the gas production can be used as mathematical models applied in the modeling of production and import of natural gas in Brazil

Keywords: Model of Malthus. Model of Verhust. Mathematical modeling. Natural gas.

\section{INTRODUÇÃO}

Mundialmente o uso do gás natural está em crescimento no setor industrial 
(Kim, 1999) proporcionando um aumento do uso no setor de transporte e na geração de energia elétrica. Sua utilização nas usinas termelétricas trouxe vantagens financeiras e ambientais, diversificação do uso de combustíveis, intensificou questões de segurança e contribuiu para desregulamentação mercadológica e crescimento econômico mundial.

Segundo o Department of Energy, o consumo mundial de gás natural deverá aumentar a uma taxa de 2,3\% ao ano até 2025 (DOE, 2008). No Brasil, com a crescente participação do gás natural na matriz energética, principalmente após a crise no setor elétrico em 2001, este aumento também será observado.

O fornecimento de gás natural no país é dependente da Bolívia desde o final da construção do gasoduto, em 1999. Essa dependência chegou a 50 \% em 2006 (SantAna, 2008), o que acarretou em problemas no abastecimento devido à questões políticas enfrentadas pela Bolívia em 2006. Esses problemas causaram uma crise no abastecimento de gás no Brasil, o que levou a Petrobras a rever a dependência do Brasil ao gás boliviano para 22 \% em 2016 (SANTANA, 2008).

O governo federal brasileiro, por meio de políticas de desenvolvimento, pretende ampliar a participação do gás natural na matriz energética de $2 \%$ para $12 \%$ nos próximos dez anos (LOURENÇO, 2005). Para tanto, deverá haver um aumento significativo na produção de gás natural, que poderá atingir aproximadamente 95 milhões de metros cúbicos por dia em 2010 (PDE, 2008). Porém, as reservas brasileiras de gás natural apresentam duas características principais: $80 \%$ são de gás associado e $55 \%$ estão situadas em águas profundas, o que significa que a oferta de gás natural é influenciada e muito dependente da produção de petróleo.

O aumento da capacidade de abastecimento de energia elétrica no Brasil só vai ocorrer com a geração térmica com uso do gás natural (Moreira, 2007). A falta de investimentos em infraestruturas de energia elétrica pelos governos estaduais e federais e a volta do crescimento do PIB em torno de 4\% ao ano até 2012 podem indicar um cenário de escassez de energia elétrica entre os anos de 2010 e 2012. Essa escassez de energia pode viabilizar contratos flexíveis para aquisição de gás natural para utilização em geração distribuída (GD) que a partir de 2013 receberá 
uma política de incentivos do governo federal (Wright, 2009).

\section{MÉTODOS}

O estudo foi realizado a partir dos dados da produção e importação de gás natural, provenientes do Balanço Energético Nacional do Ministério de Minas e Energia (MME, 2009).

Os modelos matemáticos já são aplicados na previsão e equilíbrio do mercado de gás natural no mundo (ZHUANG, 2008). O modelo matemático adotado para previsão da produção de gás natural no Plano Decenal de Expansão de Energia (PDE 2008-2017) foi desenvolvido pela Coordenação de Projetos, Pesquisa e Estudos Tecnológicos (Coppetec) (FERREIRA, 2007). Pela dificuldade de obtenção de dados necessários para uma simulação de fluxo de reservatórios, tais como dados petrofísicos, características de fluidos e parâmetros de fluxo (pressões e vazões), o modelo desenvolvido por Ferreira (2007) utiliza três etapas: crescimento, produção estabilizada e produção em declínio. As fases de crescimento e patamar são modeladas por uma função linear, enquanto a fase de declínio é modelada por uma função exponencial ou hiperbólica. Esse modelo matemático desenvolvido pela Coppetec permite, por meio de um programa computacional, registrar o ritmo de produção de gás natural, quando ocorrem eventos de incremento ou decremento de produção, tais como entrada de novas plataformas em funcionamento ou fechamento de poços.

A dinâmica de populações (RONEY, 2006) trata das variações, no tempo e no espaço, das densidades e tamanhos das populações. O estudo da dinâmica populacional não se restringe somente à compreensão da variação do número de indivíduos de uma determinada população, mas também no estudo do controle biológico de pragas, estratégias de pesca e crescimento de cidades. Os modelos que tratam do crescimento populacional são os modelos de Malthus e Verhulst. Esses dois modelos tratam do crescimento populacional e a aplicação dos mesmos é possível no estudo da produção e importação de gás natural, pois os dados 
apresentados no balanço energético mostram características de crescimento populacional, isto é, dados com crescimento exponencial, e regiões de estabilização no caso da importação de gás natural.

Os modelos aplicados neste trabalho são Malthus e Verhust. Estes dois modelos, apesar de tratarem do crescimento populacional, têm viabilidade de aplicação ao estudo de produção e importação de gás natural, pois os dados apresentados no balanço energético mostram características de crescimento populacional, isto é, dados com crescimento exponencial, e regiões de estabilização no caso da importação de gás natural (Tabelas 1 e 2).

Tabela 1: Produção de gás natural

\begin{tabular}{lccc}
\hline Ano & Produção $\left(10^{6} \mathrm{~m}^{3}\right)$ & Ano & Produção $\left(10^{6} \mathrm{~m}^{3}\right)$ \\
\hline 1970 & 1.264 & 1990 & 6.279 \\
1971 & 1.178 & 1991 & 6.597 \\
1972 & 1.241 & 1992 & 6.976 \\
1973 & 1.180 & 1993 & 7.355 \\
1974 & 1.488 & 1994 & 7.756 \\
1975 & 1.625 & 1995 & 7.955 \\
1976 & 1.642 & 1996 & 9.156 \\
1977 & 1.808 & 1997 & 9.825 \\
1978 & 1.933 & 1998 & 10.788 \\
1979 & 1.899 & 1999 & 11.898 \\
1980 & 2.205 & 2000 & 13.283 \\
1981 & 2.475 & 2001 & 13.998 \\
1982 & 3.030 & 2002 & 15.568 \\
1983 & 4.013 & 2003 & 15.792 \\
1984 & 4.902 & 2004 & 16.971 \\
1985 & 5.467 & 2005 & 17.699 \\
1986 & 5.686 & 2006 & 17.706 \\
1987 & 5.781 & 2007 & 18.152 \\
1988 & 6.076 & 2008 & 21.593 \\
1989 & 6.105 & & \\
\hline
\end{tabular}

Fonte: MME, 2009. 
A Tabela 1 apresenta os dados da produção de gás natural e a Tabela 2 apresenta os dados da importação de gás natural pelo Brasil. Esses dados foram ajustados para utilizá-los nas equações diferenciais que descrevem os modelos de crescimento populacional. Alguns parâmetros das equações diferenciais foram linearizados e outros obtidos por meio do método dos mínimos quadrados.

Tabela 2: Importação de gás natural

\begin{tabular}{cc}
\hline Ano & Importação $\left(10^{6} \mathrm{~m}^{3}\right)$ \\
\hline 1999 & 400 \\
2000 & 2.211 \\
2001 & 4.608 \\
2002 & 5.269 \\
2003 & 5.055 \\
2004 & 8.086 \\
2005 & 8.998 \\
2006 & 9.789 \\
2007 & 10.334 \\
2008 & 11.314 \\
\hline Fonte: MME, 2009.
\end{tabular}

Para utilizar os modelos aplicados à dinâmica populacional na produção e importação de gás natural foi considerado, no modelo de Malthus, que a taxa de crescimento da população $r$ é a taxa de crescimento da produção de gás e $\alpha$ a taxa de crescimento média da produção $\mathrm{N}(\mathrm{t})$, conforme pode ser visto nas Equações $1 \mathrm{e}$ 2.

$$
\begin{aligned}
& r=\ln (1+\alpha) \quad(\text { Equação 1) } \\
& \alpha=\sqrt[t]{\frac{N_{t}}{N_{0}}}-1 \quad \text { (Equação 2) }
\end{aligned}
$$

Para o modelo de Verhulst, foi considerado que a taxa de crescimento da população $r$ é a taxa de crescimento da produção e importação de gás natural, 
sendo $r$ obtido com uso de um ajuste linear do tipo $y=b t+a$, com $b=r$. Os dados utilizados no ajuste linear do parâmetro $r$ foram os dados da Tabela 1 . O valor de $k$, que é o nível de saturação da produção e importação de gás natural, foi obtido por meio de testes com vários valores de $k$ até encontrar o menor valor para a soma dos quadrados dos desvios $S$. Toda parte computacional do trabalho foi desenvolvida utilizando o software MatLab ${ }^{\circledR}$.

\subsection{Modelo de Malthus}

O modelo de Malthus, ou modelo exponencial, é o modelo mais simples que descreve o crescimento populacional de algumas espécies. É representado por uma equação diferencial de primeira ordem que estabelece a taxa de variação da população em relação ao tempo, escrita da seguinte forma:

$$
\frac{d N}{d t}=r N(\text { Equação 3) }
$$

Onde: $\frac{d N}{d t}$ é taxa de variação da população, $r$ é a taxa de crescimento da população, conforme Equação 4:

$$
r=(1+\alpha)(\text { Equação } 4)
$$

A taxa de crescimento, $\alpha$, média da população é dada pela Equação 5:

$$
\alpha=\sqrt[t]{\frac{N_{t}}{N_{0}}}-1 \text { (Equação 5) }
$$

$N_{t}$ é a população após um período de $t$ anos em relação a população inicial $N_{0}$

A Equação 3 apresenta a seguinte solução:

$$
N(t)=N_{0} e^{r t}
$$

Onde: $N_{0}$ é a população inicial $\mathrm{N}(0)=\mathrm{N}_{0}$.

Como o modelo exponencial não apresenta limites para o número de 
indivíduos de uma população, fica claro que esse modelo não representa um quadro realístico de crescimento populacional. O modelo exponencial pode ser realístico para o crescimento de algumas populações durante um intervalo de tempo relativamente curto.

\subsection{Modelo de Verhulst}

O modelo de Verhulst, ou modelo logístico, supõe que uma população deverá crescer até um limite máximo, isto é, a população tende a se estabilizar. Essa estabilidade da população no modelo de Verhulst está relacionada com a capacidade de suporte do meio que esta população vive. A equação diferencial para este modelo é representada pela Equação 6:

$$
\frac{d N}{d t}=r N\left(1-\frac{N}{k}\right)(\text { Equação } 6)
$$

Onde: $r$ é a taxa de crescimento da população e k é o nível de saturação da população.

A solução da Equação 6 é obtida pelo método de variáveis separáveis (Zill, 2003), juntamente com o uso da técnica de integração de frações parciais, sendo:

$$
N(t)=\frac{k N_{0}}{N_{0}+\left(k-N_{0}\right) e^{-r t}}
$$

Onde: $\mathrm{N}(0)=\mathrm{N}_{0}$ sendo que $N_{0}$ não é igual a zero nem igual a $k$.

\section{RESULTADOS E DISCUSSÕES}

Serão apresentados a seguir e separadamente, os resultados obtidos para produção e importação de gás natural. Em cada conjunto de dados, produção e importação de gás natural, aplicaram-se os dois modelos estudados para verificar qual modelo melhor descreve os dados de importação e produção de gás natural. No final da seção uma comparação com o modelo utilizado no Plano Decenal de 
Expansão de Energia será feita.

\subsection{Produção de gás natural}

Os resultados obtidos para os modelos exponencial e logístico com os dados da Tabela 1 são apresentados a seguir. Para o modelo exponencial a taxa de crescimento média da produção $\alpha$ é dada por:

$\alpha=\sqrt[37]{\frac{18.152}{1.500}}-1$, com $\mathrm{N}_{0}=1.500 \times 10^{6} \mathrm{~m}^{3}$ de gás. O valor de $N_{0}$ é a condição inicial da Equação 3.

A Figura 1 apresenta o resultado do modelo exponencial para os dados da produção de gás.



Figura 1: Produção de gás natural em função do tempo com modelo exponencial 
Para o modelo logístico apresentado na Figura 2, o valor de $k$ utilizado foi de 49500 que apresentou um valor de $S$ de 0,7322 .

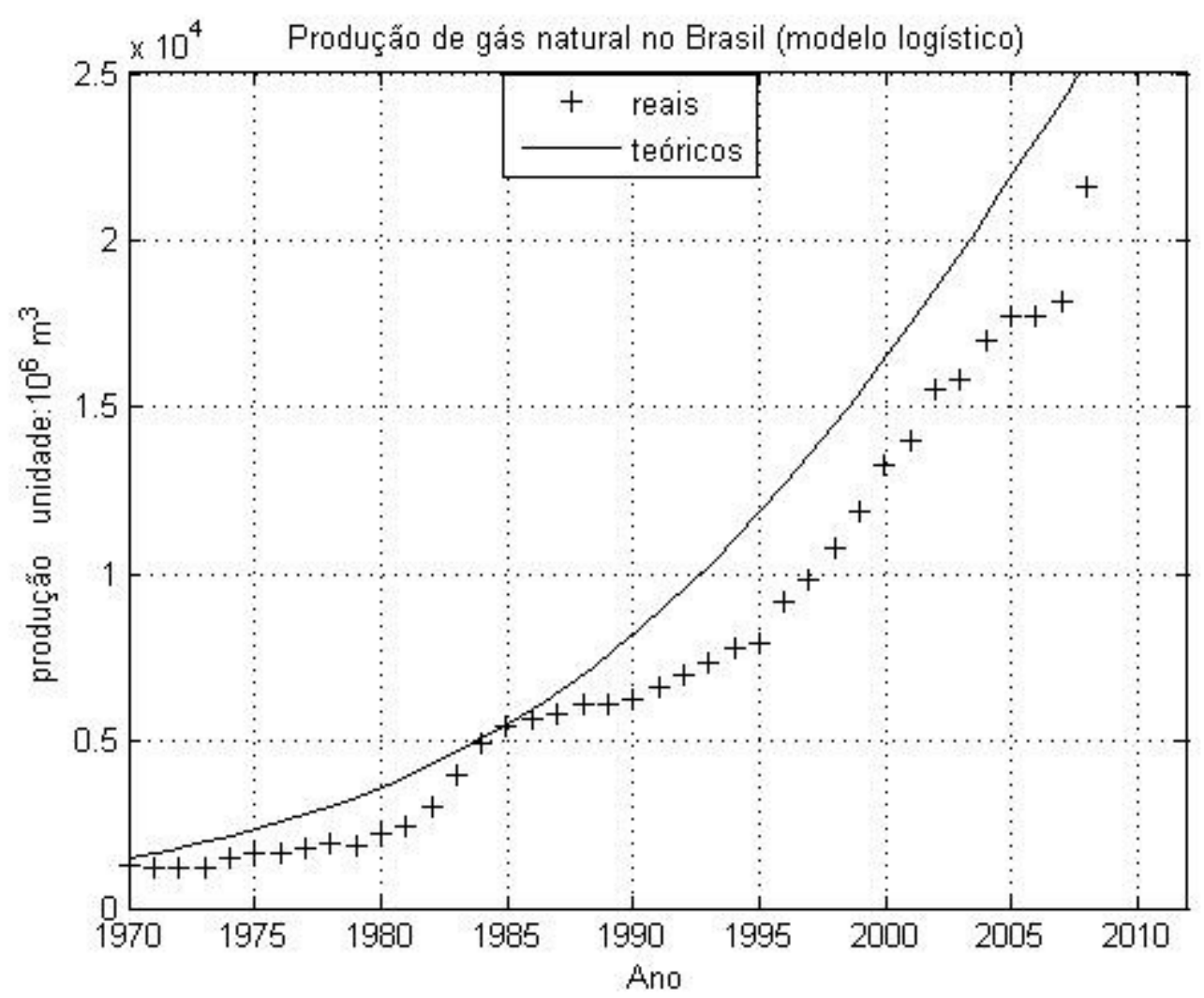

Figura 2: Produção de gás natural em função do tempo com modelo logístico

Os resultados obtidos mostraram que com os ajustes feitos na condição inicial $\left(\mathrm{N}_{0}\right)$ do modelo exponencial ou de Malthus apresentaram resultados mais adequados para a modelagem dos dados da produção de gás natural, conforme descrito na Figura 2. A maior diferença entre os dados apresentados na Tabela $1 \mathrm{e}$ os resultados do modelo está entre os anos de 1983 e 1990. O resultado apresentado na Figura 1 é semelhante ao resultado apresentado por SantAna (2008) que utilizou uma curva logística para modelar o gráfico.

\subsection{Importação de gás natural}


O Brasil começou a importar gás natural em 1999, com isso, ficaram poucos pontos para aplicar o modelo exponencial ou o modelo logístico, conforme Tabela 2. O mesmo procedimento feito para a produção de gás natural foi aplicado para a importação de gás natural. Para o modelo exponencial o valor de $\alpha$ é dado por: $\alpha=\sqrt[8]{\frac{10.334}{3.200}}-1$, com $\mathrm{N}_{0}=3200 \times 10^{6} \mathrm{~m}^{3}$ de gás.

Já o valor de k utilizado no modelo logístico foi de 11930 que apresentou um valor de $S=2,0964$.

Os resultados obtidos para a importação de gás natural são apresentados a seguir.

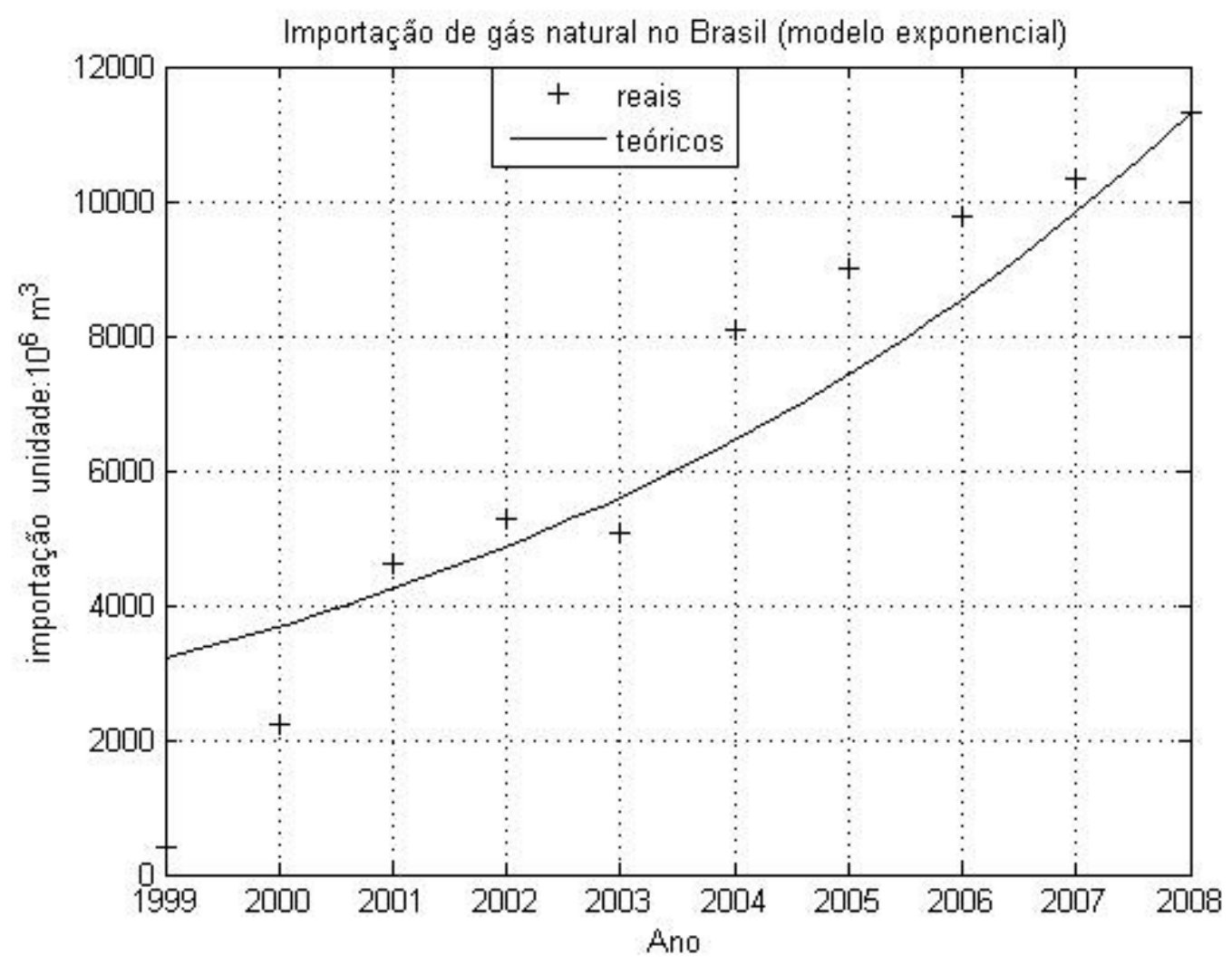

Figura 3: Importação de gás natural em função do tempo com modelo exponencial

Para o modelo logístico apresentado na Figura 4 , o valor da constante $k$ foi 
de $10780 \times 10^{6} \mathrm{~m}^{3}$ de gás e $\mathrm{N}_{0}=1000 \times 10^{6} \mathrm{~m}^{3}$.

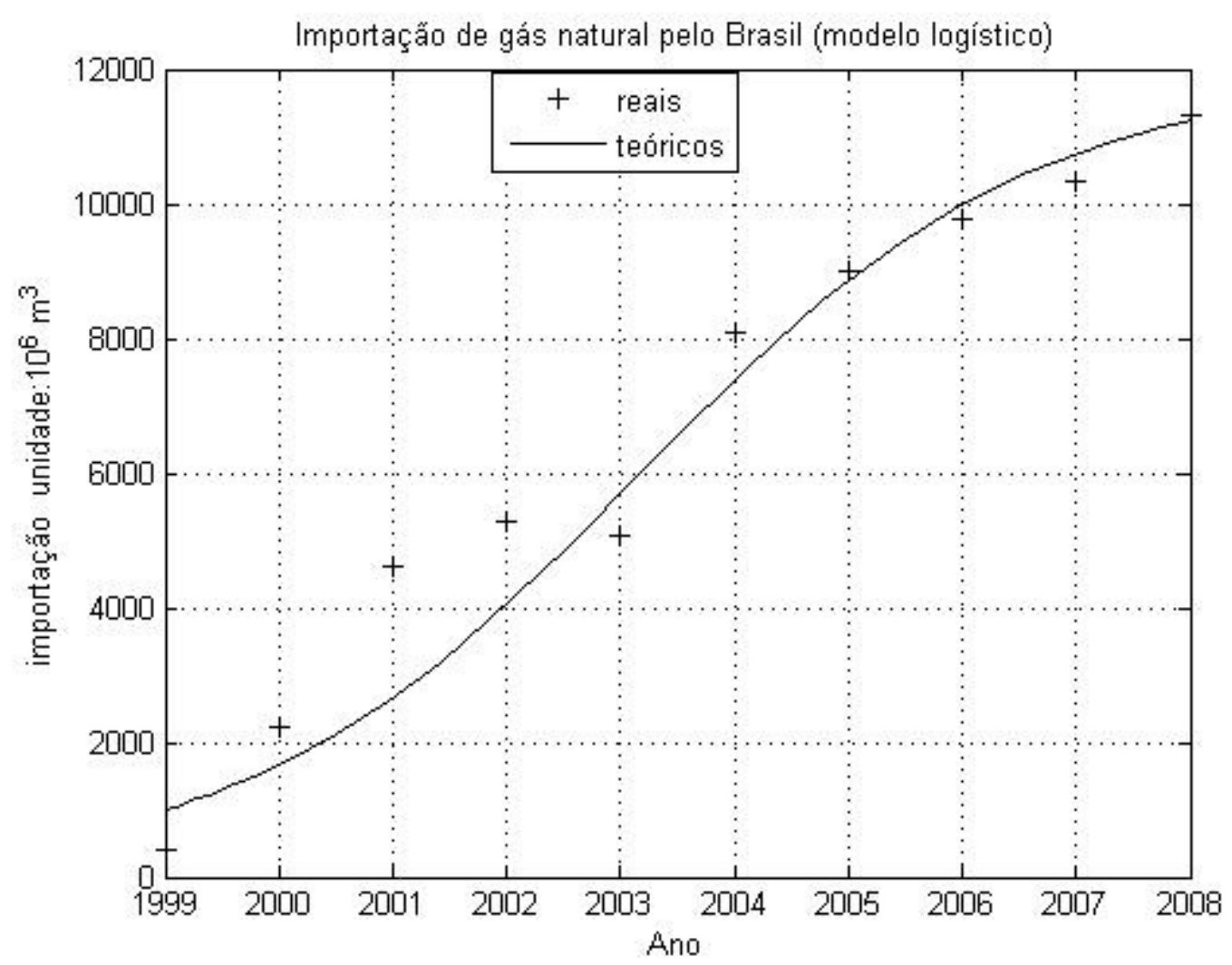

Figura 4: Importação de gás natural em função do tempo com modelo logístico

Para a importação de gás natural, apesar dos poucos pontos analisados devido ao início da importação no Brasil dá-se somente em 1999, o modelo logístico ou de Verhulst apresentou resultados mais adequados aos fins, conforme descrito na Figura 4. $O$ gráfico apresentado na Figura 4 mostra que a importação de gás tende a se estabilizar, o que foi descrito por Santana (2008), principalmente com a diminuição da dependência do gás boliviano.

\subsection{Projeção da produção e importação de gás natural até 2017 com uso dos modelos de dinâmica populacional}

Nos gráficos e tabelas a seguir, estão as previsões de produção de gás 
natural apresentadas no PDE 2008 - 2017.

A Tabela 3 e a Figura 5 representam a previsão de produção diária de gás nacional até 2017. Analisando apenas a produção total (PT), prevê-se uma tendência crescente até 2010, quando atingirá um patamar de produção de 95 milhões de metros cúbicos por dia. Esse patamar será mantido até 2014, ano que se inicia um novo crescimento até 2016, quando atingirá um patamar de 140 milhões de metros cúbicos por dia.

Tabela 3: Previsão da produção de gás natural em $\mathrm{MM} \mathrm{m}$ ºlia no período de 2007 -2017

\begin{tabular}{cccccccccccc}
\hline Recursos & 2007 & 2008 & 2009 & 2010 & 2011 & 2012 & 2013 & 2014 & 2015 & 2016 & 2017 \\
\hline RND & 0 & 0 & 0 & 0 & 0 & 0 & 0 & 10,082 & 22,682 & 30,241 & 31,5 \\
RC & 0 & 0 & 0 & 0,576 & 0,784 & 2,022 & 4,699 & 8,694 & 11,372 & 17,814 & 20,493 \\
RT & 49,766 & 60,971 & 77,475 & 94,778 & 94,231 & 95,142 & 96,810 & 94,920 & 95,186 & 91,446 & 88,151 \\
TOTAL & 49,766 & 60,971 & 77,475 & 95,354 & 95,015 & 97,164 & 101,509 & 113,696 & 129,24 & 139,501 & 140,144
\end{tabular}

Fonte: PDE, 2008.

onde : RND são recursos não-descobertos nos campos a descobrir;

RC são recursos contingentes nos campos em estágio de avaliação;

RT são reservas totais nos campos em desenvolvimento ou em produção. 


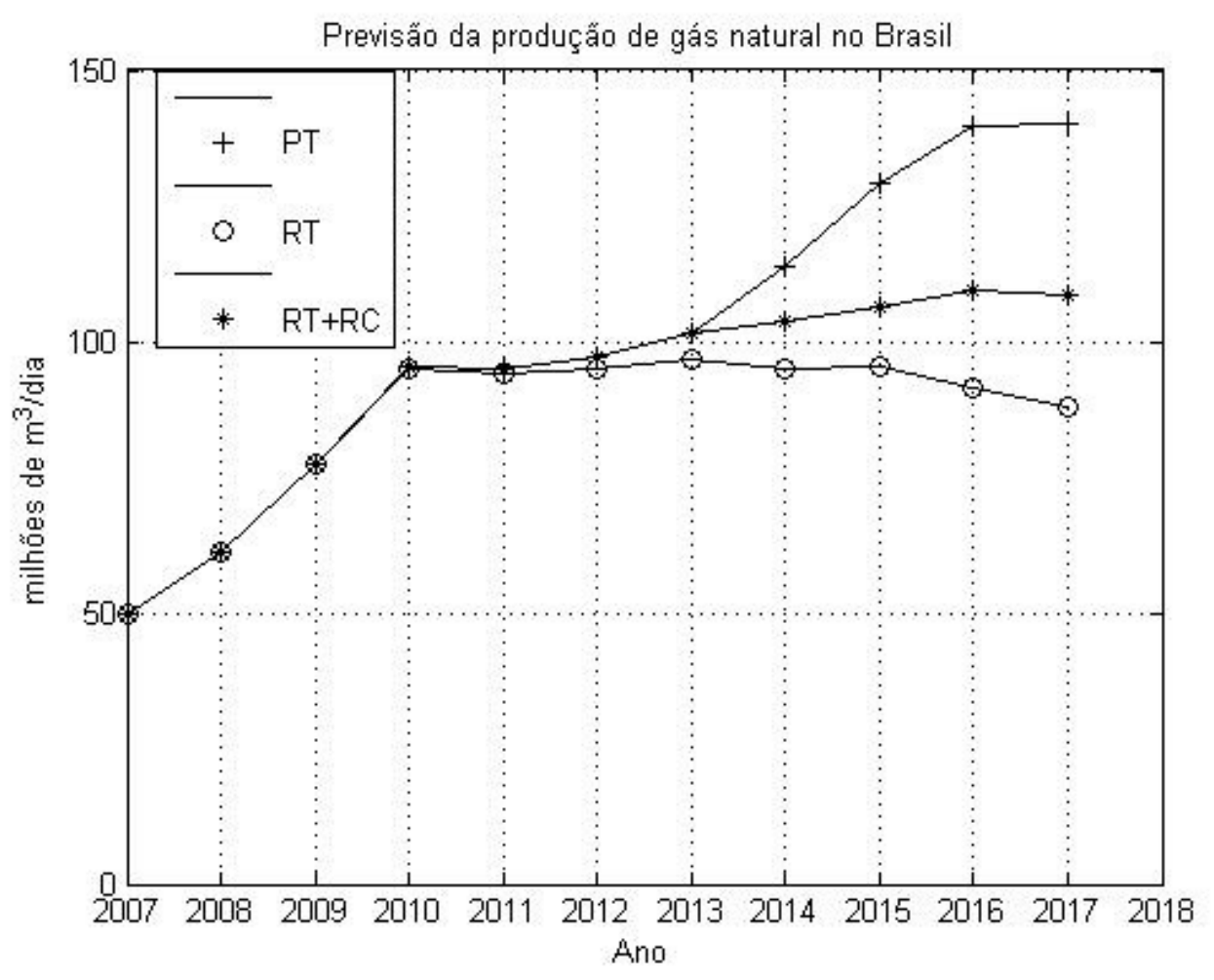

Figura 5: Previsão da produção de gás natural em função do tempo

A tabela 5 e a Figura 6 apresentam a previsão de importação de gás natural, provenientes do gasoduto da Bolívia e eventualmente da Argentina. Já a oferta via gás natural liquefeito (GNL), refere-se aos projetos de importação de gás para as regiões Nordeste e Sudeste do país.

Tabela 4: Capacidade de oferta de gás natural - Importado $\left(\mathrm{MMm}^{3} / \mathrm{dia}\right)$

\begin{tabular}{ccccccccccc}
\hline Origem & 2008 & 2009 & 2010 & 2011 & 2012 & 2013 & 2014 & 2015 & 2016 & 2017 \\
\hline Gasodutos - S-SE-CO & 29,1 & 30,1 & 30,1 & 30,1 & 30,1 & 30,1 & 30,1 & 30,1 & 30,1 & 30,1 \\
GNL - Nordeste & 6 & 6 & 6 & 6 & 6 & 6 & 6 & 6 & 6 \\
GNL - S-SE-CO & 14 & 14 & 14 & 14 & 14 & 14 & 14 & 14 & 14 \\
GNL - Novos & & & & & & 11 & 11 & 15 & 15 & 15
\end{tabular}




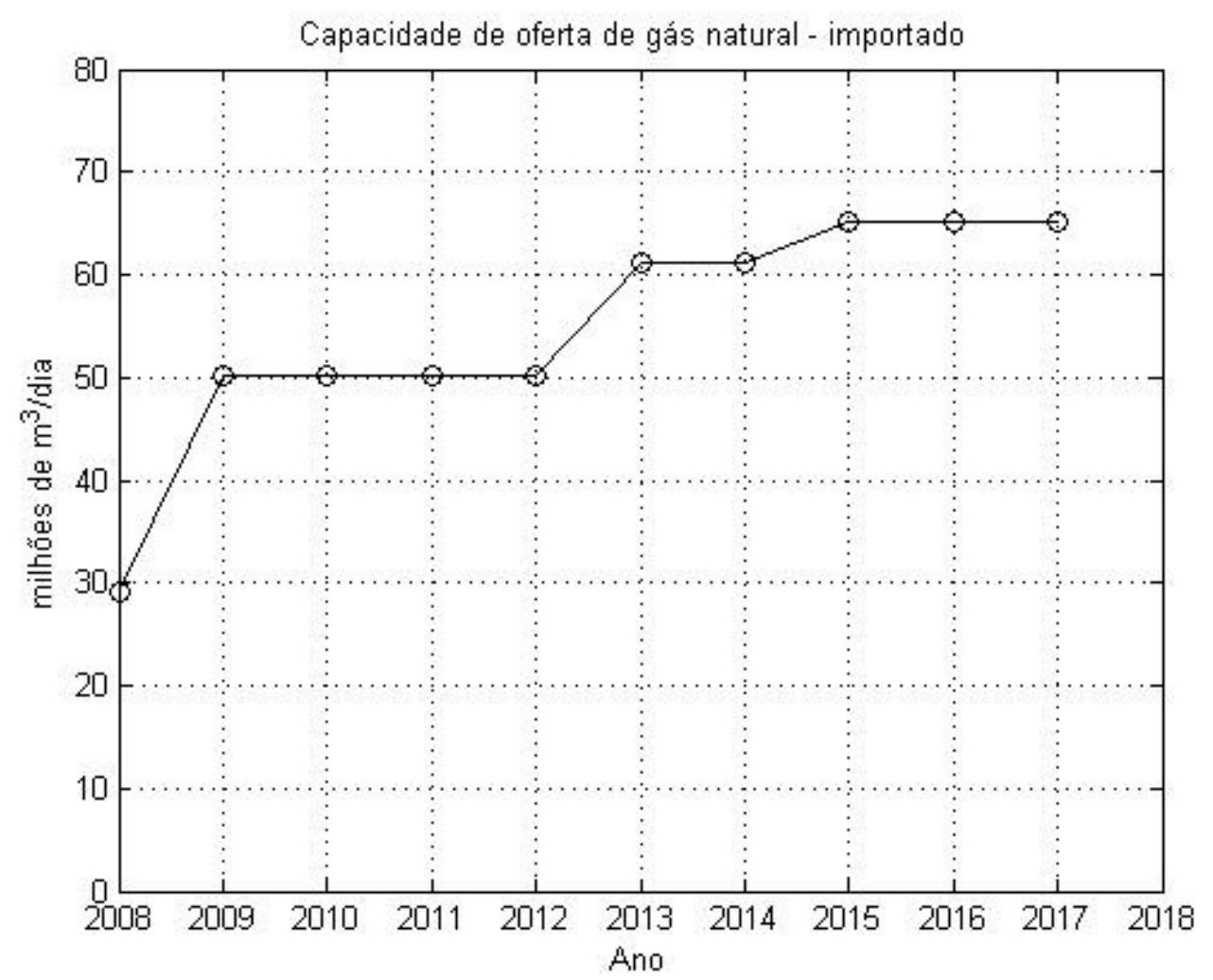

Figura 6: Previsão de importação de gás natural

A Tabela 5 e a Figura 7 apresentam as contribuições do gás associado (GA) e do gás não-associado (GNA) para as previsões de produção a partir dos recursos descobertos. A produção de gás associado é maior, porém a relação (GA/GNA) começa a alterar-se a partir de 2010, tornando-se praticamente equivalentes em 2017. 
Tabela 5: Previsão da produção de gás natural em milhões de metros cúbicos por dia no período de 2007 -2017, a partir de recursos descobertos

\begin{tabular}{cccccccccccc}
\hline Gás & & & & & & & & & & & \\
Natural & 2007 & 2008 & 2009 & 2010 & 2011 & 2012 & 2013 & 2014 & 2015 & 2016 & 2017 \\
GA & 37,877 & 42,088 & 49,448 & 49,913 & 49,447 & 51,875 & 53,602 & 53,889 & 53,153 & 56,915 & 57,083 \\
GNA & 11,888 & 18,883 & 28,027 & 45,441 & 45,568 & 45,29 & 47,907 & 49,725 & 53,405 & 52,344 & 51,56 \\
Total & 49,766 & 60,971 & 77,475 & 95,354 & 95,015 & 97,164 & 101,509 & 103,614 & 106,558 & 109,259 & 108,644
\end{tabular}

Fonte: PDE, 2008.

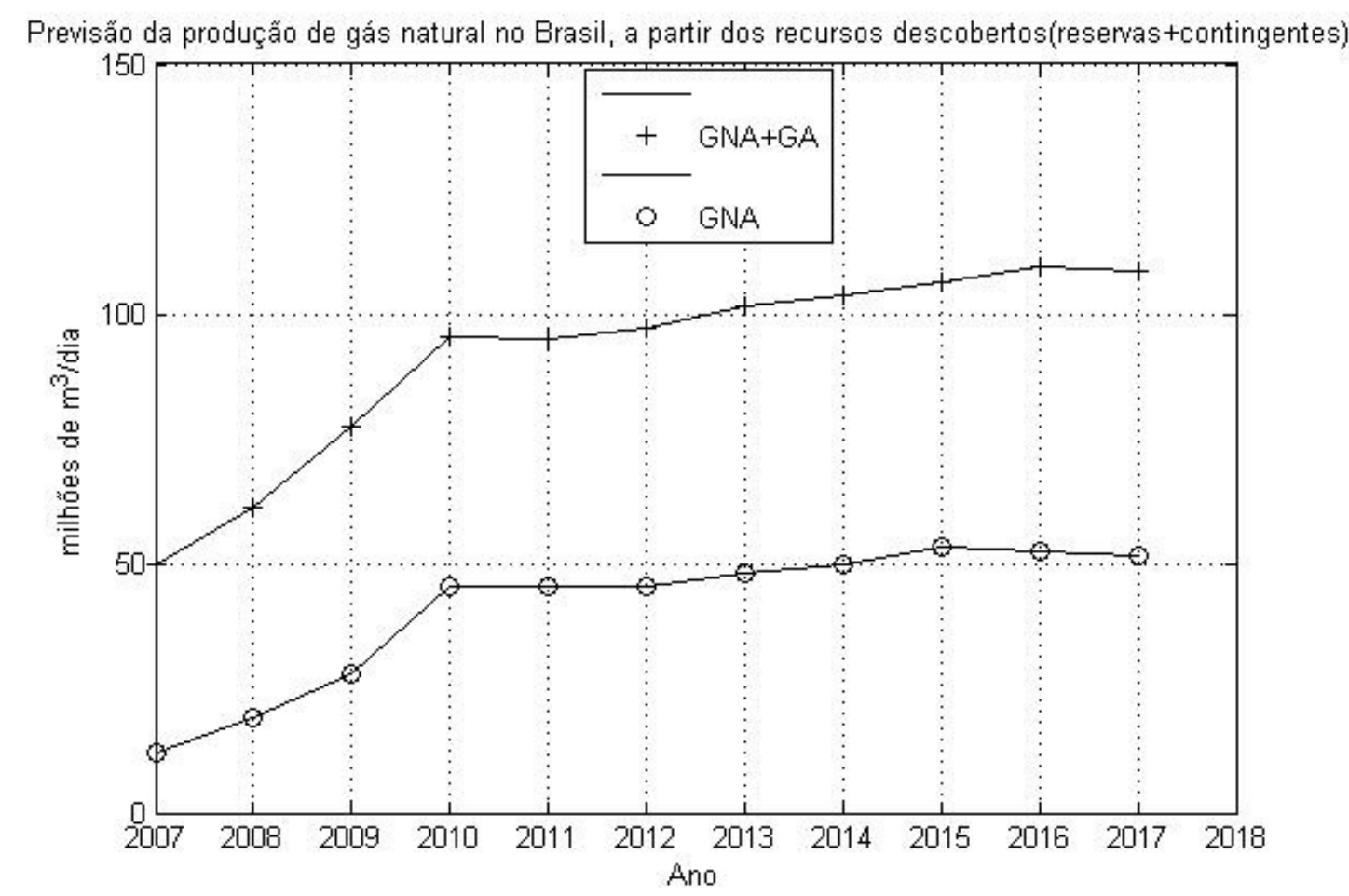

Figura 7: Previsão de produção de gás natural em função do tempo a partir dos recursos descobertos

Com base nos resultados nas seções 3.1 e 3.2 , verificou-se que o melhor modelo que descreve a produção de gás natural é o modelo exponencial e o melhor modelo que descreve a importação de gás natural é o modelo logístico. A partir desses resultados foi possível fazer uma previsão da produção e importação de gás natural até 2017. A Figura 8 mostra a previsão da importação de gás natural até 2017 a partir do modelo logístico de Verhulst. Comparando os resultados da Tabela 4 
sem a importação de GNL com os resultados do modelo de Verhulst, Figura 9, verificou-se que os resultados estão próximos. O resultado a partir do modelo logístico apresentou uma importação de 32,6 milhões de metros cúbicos por dia em 2015 e o valor da Tabela 4 para o mesmo período foi de 30,1 milhões de metros cúbicos por dia.

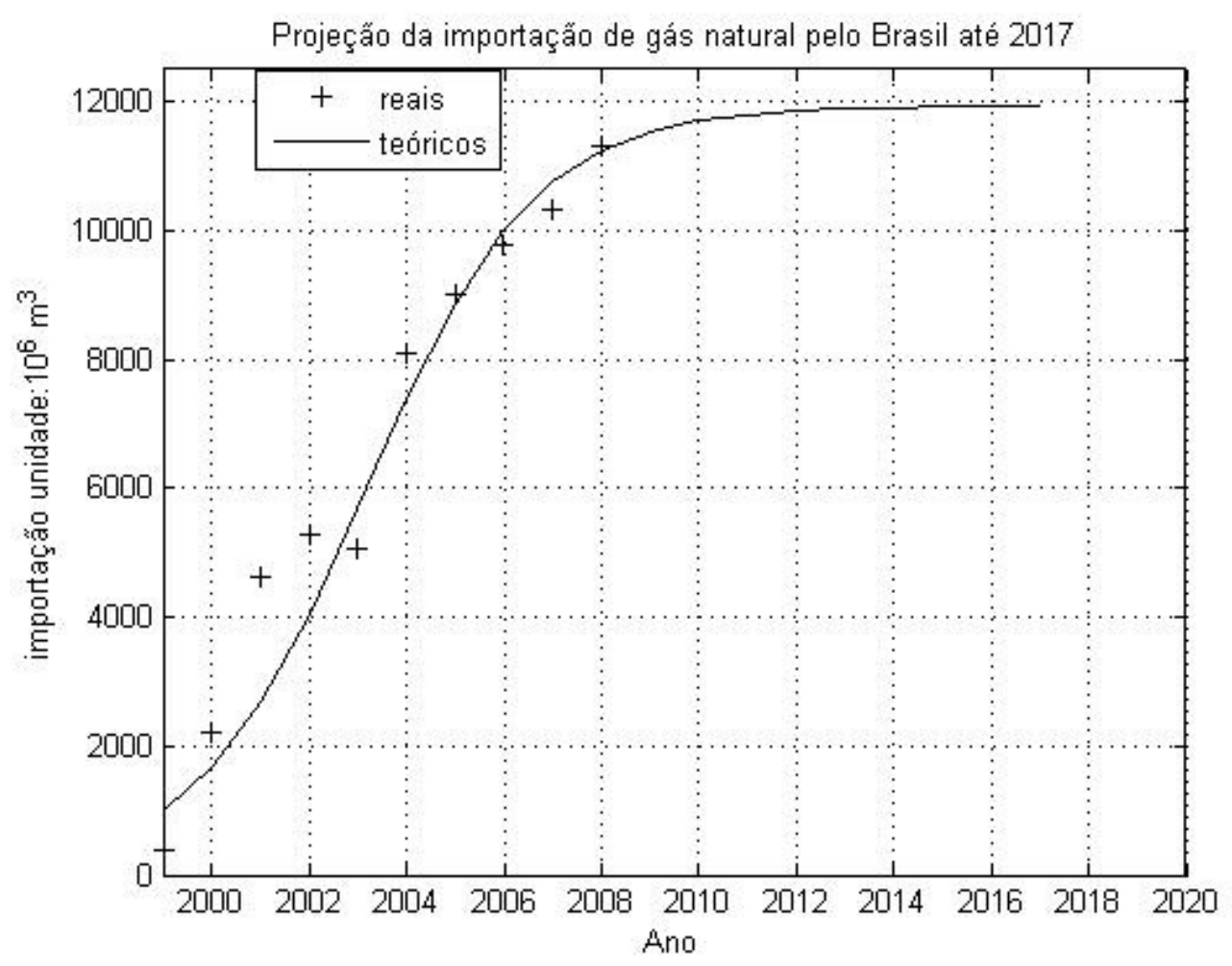

Figura 8: Projeção da importação de gás natural em função do tempo com modelo logístico 


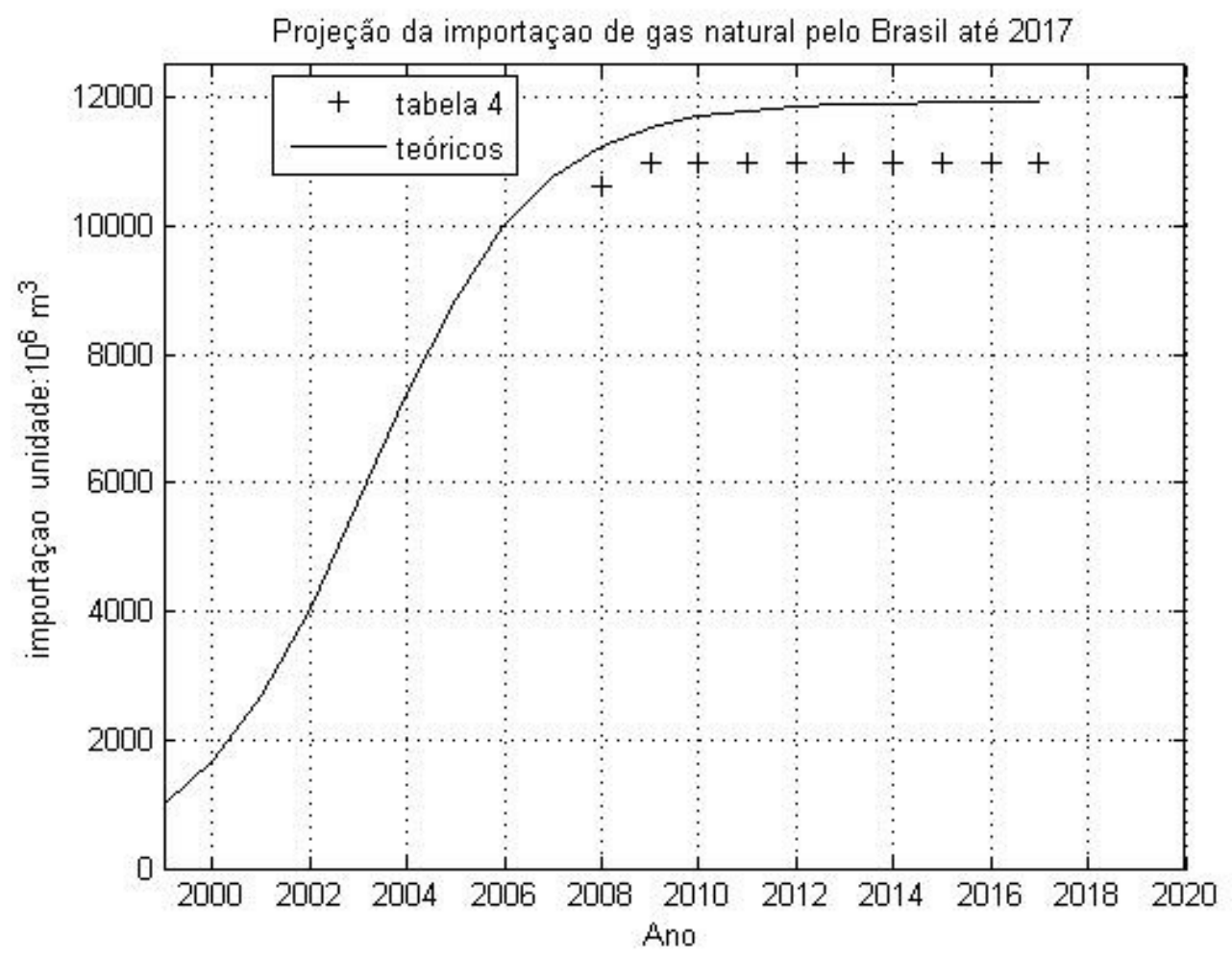

Figura 9: Gráfico da comparação dos dados da tabela 4 com modelo logístico

A Figura 10 mostra a previsão da produção de gás natural até 2017 a partir do modelo logístico de Malthus. Comparando os resultados da Tabela 3, levando em consideração somente as reservas totais, com os resultados apresentados do modelo de Malthus, Figura 11, foram verificados que dois pontos (2008 e 2015) estão próximos. O resultado a partir do modelo exponencial apresentou uma produção de 96,8 milhões de metros cúbicos por dia em 2015 e o valor da Tabela 3 para o mesmo período foi de 95,186 milhões de metros cúbicos por dia. 


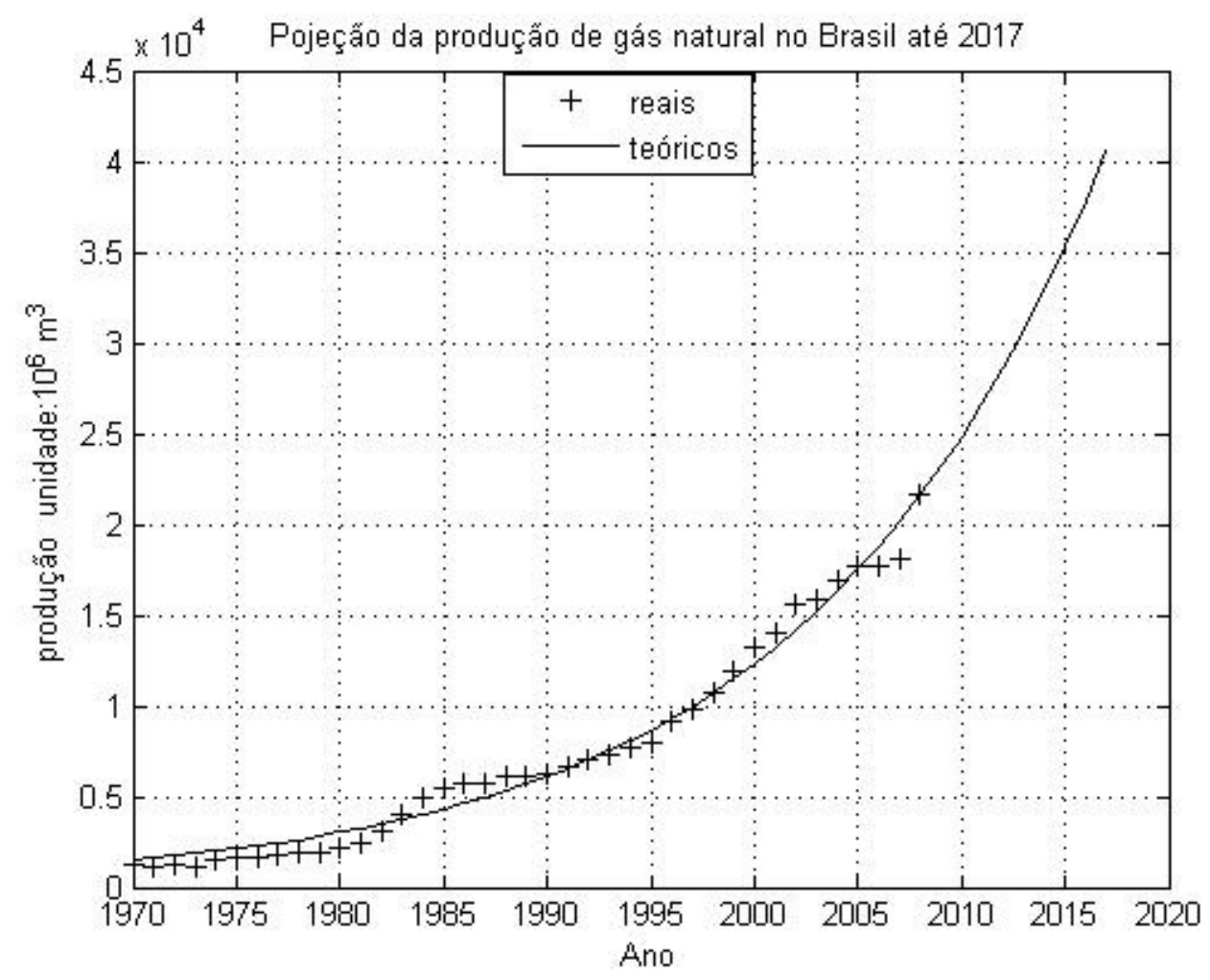

Figura 10: Projeção da produção de gás natural em função do tempo com modelo exponencial

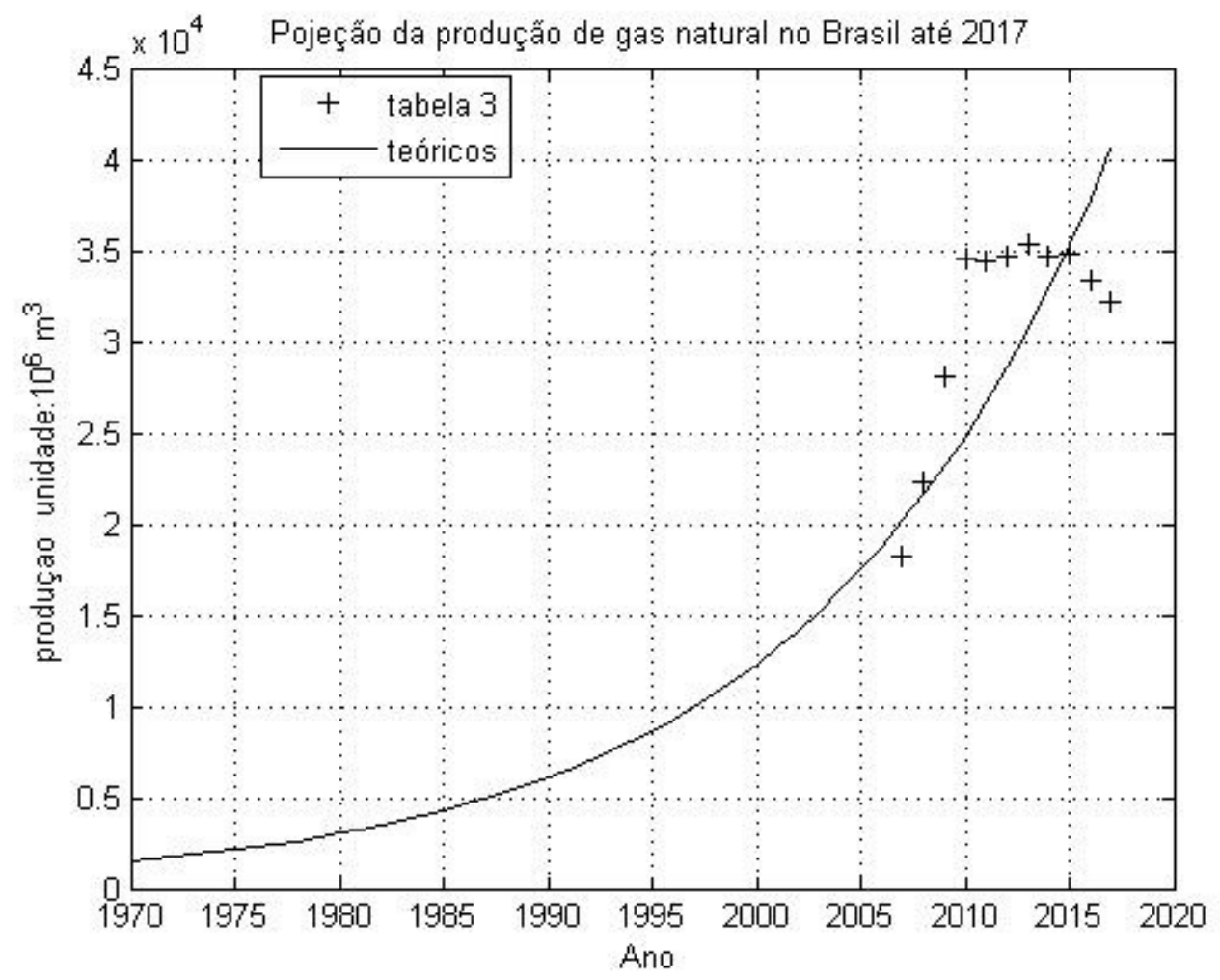

Revista Produção Online, v.10, n.3, p. 698-717, set., 2010 
Figura 11: Comparação dos dados da Tabela 3 com modelo exponencial

Comparando os resultados deste trabalho que utilizou somente os dados publicados no BEM - 2008 com os dados apresentados no PDE - 2008 que utilizou uma análise complexa para obter os resultados da previsão da produção e importação de gás natural até 2017, verificou-se que os modelos de dinâmica populacional apresentam resultados para previsão da produção e importação de gás natural até 2017 satisfatórios em relação aos resultados apresentados no PDE 2008.

\section{CONCLUSÃO}

Com base nos resultados obtidos o modelo de Malthus descreve melhor os dados da produção de gás natural e o modelo de Verhulst descreve melhor os dados da importação de gás natural. Portanto, os resultados iniciais apresentados neste trabalho mostram que os modelos utilizados no estudo de dinâmica populacional podem ser utilizados no estudo da produção e importação de gás natural no Brasil.

\section{REFERÊNCIAS}

DEPARTMENT OF ENERGY (DOE). Annual energy outlook. New York: EIA, 2008. Disponível em:< http://www.eia.doe.gov>. Acesso em: 15 dez. 2008.

FERREIRA, V. J. M., Manual de utilização do sistema interativo de previsão de produção e de reservas. Rio de Janeiro: EPE, 2007.

KIM, T. Y., LEE J. D., PARK, Y. H. International comparisons of productivity and its determinants in the natural gas industry. Energy Economics, v. 21, p. 273-293, 1999.

LISE, W., HOBBS, B. F. Future evolution of the liberalized European gas market: Simulation results with a dynamic model. Energy, v. 33, p. $989-1004,2008$.

MINISTÉRIO DE MINAS E ENERGIA (MME). Balanço energético nacional 2008. 
Brasília: EPE, 2009.

MOREIRA, T. R., VELOSO, L., REGRA, A. O desafio do gás natural: o problema da segurança do abastecimento. In: CONGRESSO BRASILEIRO DE REGULAÇÃO, 5., 2007. Recife. Anais... Recife, 2007.

EMPRESA DE PESQUISA ENERGÉTICA (EPE). Plano decenal de expansão de energia Rio de Janeiro: EPE, 2009.

RONEY R. N. Dinâmica de populações: um breve histórico. In: BIENAL DA SBM IME/UFG, 3., 2006. Goiânia: Anais eletrônicos. Goiânia, 2006.

Disponível em:< http://www.ime.ufg.br/bienal/2006/poster/roney.pdf $>$. Acesso em: 15 dez. 2008.

SANTANA, P. H. M., JANNUZZI, G. M., BAJAY, S. V. Developing competition while building up the infructure of brazilian gas industry. Energy Policy, v. 37, p. 308-317, 2009.

LOURENÇO, S. R., TAMBOURGI, E. B. Gás natural: perspectivas e utilização. Exacta, São Paulo, v. 3, p. 63-70, 2005.

ZHUANG, J., GRABRIEL, S. A. A complementarity model for solving stochastic natural market equilibria. Energy Economics, v. 30, p. $113-147,2008$.

ZILL, D. G., CULLEN, M.I R. Equações diferenciais. v.1. 2. ed. São Paulo: Person, 2003.

WRIGHT, J. T. C., CARVALHO, D. E. C., GIOVINAZZO R. S. Tecnologias disruptivas de geração distribuída e seus impactos futuros sobre empresas de energia. RAl - Revista de Administração e Inovação, São Paulo, v. 6, n. 1, p. 108125, 2009.

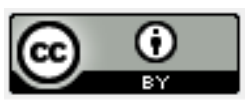

Artigo recebido em 26/04/2010 e aceito para publicação em 14/08/2010. 